\title{
Serum antinucleosome-specific antibody as a marker of autoimmunity in children with autism
}

\author{
Laila Yousef AL-Ayadhi ${ }^{1}$ and Gehan Ahmed Mostafa, ${ }^{1,2^{*}}$
}

\begin{abstract}
Background: Increasing evidence of autoimmune phenomena in some individuals with autism could represent the presence of altered or inappropriate immune responses in this disorder. The role of the nucleosome in the induction of antibody response in some autoimmune-mediated tissue damage may provide novel targets for treatment. Due to the paucity of studies investigating the frequency of systemic auto-antibodies in autism, we are the first to investigate the frequency of antinucleosome-specific antibodies in a group of autistic children.

Methods: Serum antinucleosome-specific antibodies were measured by ELISA in 60 autistic children, between the ages of 3 and 12 years, in comparison to 60 healthy children. Autistic severity was assessed using the Childhood Autism Rating Scale (CARS).

Results: Autistic children had significantly higher serum antinucleosome-specific antibodies than healthy children $(P<0.001)$. The seropositivity of antinucleosome-specific antibodies was found in $46.7 \%$ of autistic children. Autistic children with a family history of autoimmunity (40\%) had a significantly higher frequency of serum antinucleosome-specific antibodies (83.3\%) than patients without such a history $(22.2 \%, P<0.001)$.

Conclusions: Serum levels of antinucleosome-specific antibodies were increased in some autistic children. However, these data should be treated with caution until further investigations are performed with a larger subject population to determine whether these antibodies have a role in the induction of autoimmunity in a subgroup of autistic children. The role of immunotherapy in children with autism should be also studied.
\end{abstract}

Keywords: Antinucleosome-specific antibodies, Autism, Autoimmunity, Family history of autoimmunity

\section{Background}

A possible role of immune system abnormalities in the pathogenesis of some neurologic disorders, including autism, was postulated. Autoimmunity to the central nervous system is the most common of these abnormalities [1,2]. Brain-specific auto-antibodies were detected in the sera of many autistic children [3-10]. In addition, autoimmune disorders are increased in families of some children with autism [11-14]. There is a strong association between autism and the major histocompatibility complex for the null allele of $\mathrm{C} 4 \mathrm{~B}$ in the class III region. This results in low production of $\mathrm{C} 4 \mathrm{~B}$ protein, leading to repeated infections that play an important role in the

\footnotetext{
* Correspondence: gehan.mostafa2000@yahoo.com

'Autism Research and Treatment Center, AL-Amodi Autism Research Chair, Department of Physiology, Faculty of Medicine, King Saud University, Riyadh, Saudi Arabia

${ }^{2}$ Department of Pediatrics, Faculty of Medicine, Ain Shams University, 9 Ahmed El-Samman Street off Makram Ebaid, Nasr City 11511 Cairo, Egypt
}

development of autoimmunity [15-18]. Some autistic children have an imbalance of $\mathrm{T}$ helper (Th)1/Th2 subsets toward Th2, which are responsible for allergic response and production of autoantibodies [1].

One of the common serological hallmarks of autoimmune disorders is the presence of various autoantibodies in the sera of patients affected by these disorders [19]. The presence of abnormal levels of autoantibodies to intracellular antigens is a hallmark of several autoimmune diseases [20].

Evidence accumulated in recent years suggests that the nucleosome, the fundamental unit of chromatin and a normal product of cell apoptosis, plays a key role in some autoimmune diseases as it is a major target autoantigen for autoantibody mediated tissue lesions [21,22]. The broad antinucleosome antibody family includes: the nucleosome-specific antibodies (antinucleosome antibodies without anti ds-DNA and antihistone reactivities), 
the antinucleosome antibodies with anti ds-DNA reactivity, and the antinucleosome antibodies with antihistone reactivity [23]. Anti ds-DNA antibodies account for a minor part $(<30 \%)$ of the serum antinucleosome reactivity in lupus patients and nucleosome-specific autoantibodies are in large excess over anti ds-DNA in lupus patients [24].

Due to the paucity of studies investigating the frequency of systemic auto-antibodies in autism, we are the first to investigate the frequency of antinucleosomespecific antibodies in a group of autistic children.

\section{Methods}

\section{Study population}

This case-control study was conducted on 60 children who had classic-onset autism, over a period of 6 months from the beginning of June 2013 to the end of November 2013. The autistic group comprised 49 male and 11 female patients recruited from the Autism Research and Treatment Center, Faculty of Medicine, King Saud University, Riyadh, Saudi Arabia. Patients fulfilled the criteria for the diagnosis of autism according to the 4th edition of the Diagnostic and Statistical Manual of Mental Disorders [25]. Their ages ranged from 3 to 12 years (median $(\mathrm{IQR})=7$ (3) years). Patients who had associated neurological diseases (such as cerebral palsy and tuberous sclerosis) or metabolic disorders (such as phenylketonuria) were excluded from the study.

The control group comprised 60 age- and sex-matched apparently healthy children. They included 50 male and 10 female children. They were the healthy older siblings of the healthy infants who attend the Well Baby Clinic, King Khalid University Hospital, Faculty of Medicine, King Saud University, Riyadh, Saudi Arabia for routine followup of their growth parameters. The control children were not related to the children with autism and demonstrated no clinical findings suggestive of immunological or neuropsychiatric disorders. Their ages ranged from 3 to 12 years (median $(\mathrm{IQR})=6(4)$ years).

This study was approved by the local Ethical Committee of the Faculty of Medicine, King Saud University, Riyadh, Saudi Arabia. In addition, an informed written consent of participation in the study was signed by the parents or the legal guardians of the all studied subjects.

\section{Study measurements}

\section{Clinical evaluation of autistic patients}

This was based on clinical history taking from caregivers, clinical examination and neuropsychiatric assessment. In addition, assessment of the disease severity was done using the Childhood Autism Rating Scale (CARS) [26], which rates the child on a scale from one to four in each of fifteen areas (relating to people; emotional response; imitation; body use; object use; listening response; fear or nervousness; verbal communication; non-verbal communication; activity level; level and consistency of intellectual response; adaptation to change; visual response; taste, smell and touch response; and general impressions). According to this scale, children who have scored 30 to 36 have mild to moderate autism $(n=31)$, while those with scores ranging from 37 to 60 points have severe autism $(\mathrm{n}=29)$.

In addition, a family history of autoimmune diseases in controls and children with autism was ascertained in an identical fashion, but not in a blinded manner, by an expert rheumatologist. Parents were asked to fill out a questionnaire regarding which first- (parents and sibs) or second-degree relatives (grandparents, uncles and aunts) had received a diagnosis of specified autoimmune disorders. A list of autoimmune diseases with descriptions was provided. There was a verification of the diagnosis of autoimmune diseases via medical record review. The disorders inquired about in the questionnaire included rheumatoid arthritis, juvenile rheumatoid arthritis, systemic lupus erythematosus, insulin-dependent diabetes mellitus, rheumatic fever, vasculitis, ankylosing spondylitis, dermatomyositis, polymyositis, scleroderma, uveitis, Sjogren's syndrome, polyarteritis nodosa, Wegener's granulomatosus, Takayasu's arteritis, psoriasis, multiple sclerosis, vitiligo, myasthenia gravis, amyotrophic lateral sclerosis, Crohn's disease, ulcerative colitis, autoimmune thyroiditis, idiopathic thrombocytopenic purpura, Addison's disease, pemphigus, and Guillain-Barre syndrome. These disorders were chosen because all have a known or suspected autoimmune cause.

\section{Measurement of antinucleosome-specific antibodies}

This was performed by the ELISA technique (EUROIMMUN Medizinische Labordiagnostika AG, Germany) [27]. The samples were run randomly in a blinded fashion and they were run together, in parallel on the same run with the same internal standards. Antibodies to highly purified human nucleosomes (antinucleosomespecific antibodies), if present in diluted serum, would bind in the microwells. Washing the microwells removes unbound serum antibodies. Horseradish peroxidase conjugated anti-human IgG immunologically binds to the bound patient antibodies, forming a conjugate/antibody/ antigen complex. When substrate is washed in the presence of bound conjugate, it is hydrolyzed to form a blue color. The addition of an acid stops the reaction, resulting in a yellow end product. The intensity of this yellow color is measured photometrically at $450 \mathrm{~nm}$. The amount of color is directly proportional to the concentration of IgG antibodies present in the original sample. To increase accuracy, all samples were analyzed twice in two independent experiments to assess interassay variations and to ensure reproducibility of the observed results 
$(P>0.05)$. No significant cross-reactivity or interference was observed.

\section{Statistical analysis}

The results were analyzed by a commercially available software package (Statview; Abacus Concepts, Inc., Berkley, CA, USA). The data were non-parametric; thus, they were presented as median and interquartile range (IQR), which are between the 25 th and 75 th percentiles. A Mann- Whitney test was used for comparison between these data. A chi-square test was used for comparison between qualitative variables of the studied groups. A Spearman's rank correlation coefficient ' $r$ ' was used to determine the relationship between different variables. For all tests, a probability $(P)$ of less than 0.05 was considered significant. As data distribution was non-parametric, serum antinucleosome-specific antibodies levels were considered to be elevated if there levels were above the 95th percentile of the healthy control values (3.49 U/l).

\section{Results}

The characteristics of the study subjects are shown in Table 1.

\section{Serum levels of antinucleosome-specific antibodies in} healthy children and patients with autism

Autistic children had significantly higher serum levels of antinucleosome-specific antibodies than healthy controls, $P<0.001$ (Table 2). According to the highest cutoff value of serum antinucleosome-specific antibodies, increased serum levels of antinucleosome-specific antibodies were found in $46.7 \%$ (28/60) of autistic children.

On the other hand, there was a nonsignificant difference between serum levels of antinucleosome-specific antibodies of children with mild to moderate autism and patients with severe autism, $P=0.62$ (Table 2). Also, male and female patients with autism had no significant

Table 1 Demographic data of children with autism and healthy controls

\begin{tabular}{lcc}
\hline & $\begin{array}{c}\text { Children with autism } \\
(\mathbf{n}=\mathbf{6 0})\end{array}$ & $\begin{array}{c}\text { Control group } \\
(\mathbf{n}=\mathbf{6 0})\end{array}$ \\
\hline Age (in years) & $7(3)$ & $6(4)$ \\
Sex (male/female) & $49 / 11$ & $50 / 10$ \\
Family history of autoimmune & $24 / 60(40 \%)$ & $5 / 60(8.3 \%)$ \\
diseases & 14 & 3 \\
Rheumatoid arthritis & 4 & 1 \\
Insulin-dependent diabetes & 3 & 1 \\
mellitus & 2 & \\
Systemic lupus erythematosus & 1 & \\
Autoimmune thyroiditis & & \\
Rheumatic fever & & \\
\hline
\end{tabular}

Table 2 Serum levels of antinucleosome-specific antibodies in autistic patients and healthy children

\begin{tabular}{lcc}
\hline & $\begin{array}{c}\text { Serum antinucleosome-specific } \\
\text { antibodies } \\
\text { Median (IQR) }\end{array}$ & Z \\
\hline Healthy children $(n=60)$ & $1.7(1)$ & $(\boldsymbol{P})$ \\
Autistic patients $(n=60)$ & $2.9(2.6)$ & 5.6 \\
$\begin{array}{c}\text { Mild to moderate autism } \\
(n=31)\end{array}$ & $3.4(3)$ & 0.4 \\
Severe autism $(n=29)$ & $2.8(2.7)$ & $(0.62)$ \\
\hline
\end{tabular}

$\mathrm{IQR}$, interquartile range.

statistical difference between them in serum levels of antinucleosome-specific antibodies $(P=0.32)$. Serum levels of antinucleosome-specific antibodies had significant correlations neither with the age of autistic children $(P=0.48)$ nor with CARS $(P=0.50)$.

\section{A family history of autoimmune diseases in patients with autism and its relation to the frequency of antinucleosome-specific antibodies}

Twenty-four children with autism (40\%) had a first- or a second-degree relative with an autoimmune disease (rheumatoid arthritis in 14 patients, insulin-dependent diabetes mellitus in 4 patients, systemic lupus erythematosus (SLE) in 3 patients, autoimmune thyroiditis in 2 patients and rheumatic fever in 1 patient), Table 1 . Six out of the 24 autistic children with a family history of autoimmune disease (25\%) had a mother with an autoimmune disease (three had rheumatoid arthritis, one had SLE, one had insulin-dependent diabetes mellitus and one had autoimmune thyroiditis).

On the other hand, a family history of autoimmune diseases was found in 5 of the studied 60 (8.3\%) healthy children (rheumatoid arthritis in 3 children, SLE in 1 child and insulin-dependent diabetes mellitus in 1 child), Table 1 . None of the healthy children with a family history of autoimmune disease had a mother with such diseases. The frequency of autoimmune diseases among families of children with autism was significantly higher than normal children $(P<0.001)$.

Autistic children with a family history of autoimmunity had significantly higher frequency of serum antinucleosome-specific antibodies (83.3\%) than patients without such a history, $(22.2 \%, P<0.001)$ (Table 3$)$.

\section{Discussion}

Autoimmunity may have a role in the pathogenesis of autism. Immune system dysfunction may represent novel targets for treatment in autism [1-3]. In our series, autistic children had significantly higher serum levels of antinucleosome-specific antibodies than healthy controls $(P<0.001)$. According to the highest cut-off value of 
Table 3 The frequency of antinucleosome-specific antibodies in relation to a family history of autoimmune diseases in autistic patients

\begin{tabular}{cccc}
\hline Children with autism $(\mathbf{n}=\mathbf{6 0})$ & $\begin{array}{c}\text { Antinucleosome-specific } \\
\text { antibodies }\end{array}$ & $\mathbf{X}^{\mathbf{2}(\boldsymbol{P})}$ \\
\cline { 2 - 3 } & $\begin{array}{c}\text { Seropositive } \\
(\mathbf{n}=\mathbf{2 8})\end{array}$ & $\begin{array}{c}\text { Seronegative } \\
(\mathbf{n}=\mathbf{3 2})\end{array}$ & \\
\hline $\begin{array}{c}\text { A positive family history of } \\
\text { autoimmune diseases }(\mathrm{n}=24)\end{array}$ & $20(83.3 \%)$ & $4(16.7 \%)$ & 21.6 \\
$\begin{array}{c}\text { No family history of autoimmune } \\
\text { diseases }(\mathrm{n}=36)\end{array}$ & $8(22.2 \%)$ & $28(77.8 \%)$ & $(<0.001)$ \\
\hline
\end{tabular}

serum antinucleosome-specific antibodies, increased serum levels of these antibodies were found in $46.7 \%$ of autistic children. We could not trace data in literature regarding the frequency of antinucleosome-specific antibodies in children with autism to compare with our results.

Pathological $\mathrm{T}$ cell clones that recognize doublestranded DNA and nucleosomes further drive B cell production of anti-DNA and antinucleosome autoantibodies. Deposition of these autoantibodies within the brain and other organ systems contributes to the pathophysiology and clinical manifestations of autoimmune diseases such as SLE [28]. Complement-fixing IgG autoantibodies including anti-DNA and antinucleosome antibodies may cross the blood-brain barrier and combine with brain tissue antigens to form immune complexes that damage the neurological tissue in autistic patients [4].

The term 'nucleosome' defines a basic unit of chromatin. Each nucleosome consists of 146 base pairs of double stranded DNA, wrapped twice around a histone octamer, a protein core. A histone octamer consists of two molecules each of histones $\mathrm{H} 2 \mathrm{~A}, \mathrm{H} 2 \mathrm{~B}, \mathrm{H} 3$, and H4. In chromatin, nucleosomes are connected by 15 to 80 base pairs of linker DNA, to which histone H1 is attached. Anti-dsDNA and anti-histone antibodies belong to the nucleosome family as do antinucleosome-specific antibodies, since nucleosomes share several common epitopes with dsDNA and histones. Nucleosome-specific antibodies do not react with the individual components of the nucleosome (that is, DNA and histones) but recognize conformational epitopes resulting from interactions between the DNA and histone [29]. Nucleosomes are generated in vivo by the process of apoptosis, which is disturbed in some autoimmune diseases such as SLE. Nucleosomes are the major target autoantigens for autoantibodies mediating tissue lesions, especially glomerulonephritis in SLE [30,31]. Previous studies have reported that antinucleosome antibody reactivity is a very sensitive marker of SLE [32-37]. Similarly, it has been reported that $30 \%$ of SLE patients with high antinucleosomespecific antibody reactivity have little, if any, anti ds-DNA or antihistone reactivity [34].

The mechanisms that lead to the induction of antinucleosome specific autoantibodies in some autoimmune diseases remain obscure. In view of the prominence of nucleosomes which circulate at high levels in some autoimmune diseases such as SLE [38], it has been speculated that highly accelerated rates of apoptosis [39], and/ or abnormal sites or abnormal processing of apoptotic cells could lead to autoantibody production [40]. Also, nucleosomes may elicit the production of interleukin-6 and stimulation of lymphoproliferation and IgG synthesis by splenic B cells. This could result in a polyclonal activation that triggers both a specific (nucleosomedriven) and nonspecific antibody production [38]. Alteration of the selected parameters confirm the role of apoptosis and neuroinflammation mechanisms in the etiology of autism [41]. The apoptotic marker soluble fatty acid synthase antigen was reported to be high in Saudi children with severe autism, and can be considered an indicator of disease severity [42]. Disturbances in brain glutathione homeostasis may contribute to oxidative stress, immune dysfunction and apoptosis, particularly in the cerebellum and temporal lobe, and may lead to neurodevelopmental abnormalities in autism [43]. Thus, accelerated rates of apoptosis in autism, like in SLE, may be the possible reason behind the increased frequency of antinucleosomespecific autoantibodies in some autistic children as shown in this study. Additional investigation designed to expand on these data is warranted.

Other possible reasons behind the initiation of autoimmunity and the production of autoantibodies in some autistic children may be attributed to the exposure to some environmental cross-reacting antigens, which initiate autoimmune reactions in genetically susceptible individuals [4]. These environmental antigens include food allergies to certain peptides as casein of milk and gluten of wheat $[44,45]$, heavy metals exposure [46,47] and Hevea brasiliensis proteins in natural rubber latex [48]. In addition, infectious agents (for example, virus-induced autoimmunity) may play a causal in autism $[1,49]$.

Studies investigating the frequency of systemic antibodies in autism are very few. Seropositivity of antinuclear antibodies was reported in only $20 \%$ of 80 Egyptian children with autism, and this percentage was significantly higher than healthy children (2.5\%) [7]. Brain specific autoantibodies were reported in sera of a large proportion of children with autism [4-10]. This may be attributable to the imbalance of $\mathrm{T}$ helper (Th)1/Th2 subsets toward Th2, which are responsible for the production of antibodies and allergic response, in some children with autism [1]. In 2008, Mostafa and associates [6] reported seropositivity for antimyelin-associated glycoprotein antibodies in $62.5 \%$ of a group of 32 Egyptian autistic children between 3 and 8 years of age.

The explanation of the lower percentage of systemic antibodies (such as antinuclear antibodies and antinucleosome-specific antibodies) than the percentage 
of brain-specific autoantibodies reported by other studies [4-10] may be explained by the fact that autoimmunity in autism is organ-specific (that is, to brain) and not multisystemic. Diseases with multisystem autoimmunity (for example, SLE) have an increased frequency of systemic antibodies (such as antinuclear antibodies and antinucleosome-specific antibodies) and in these diseases, measurement of systemic antibodies is a reliable screening test [14]. Thus, testing for brain-specific autoantibodies seems to be more reliable than an antinucleosome-specific antibodies test in screening for autoimmunity in autism.

To further understand if autoimmunity could play a role in autism, we studied the frequency of autoimmune diseases in families of patients with autism in comparison to healthy children. The frequency of autoimmune disease among families of the former group (40\%) was significantly higher than that of the latter group (8.3\%). Previous research had also found an increased frequency of autoimmunity in families of children with autism compared to those of healthy and autoimmune control subjects [11-15]. In one study [11], a family history of autoimmune diseases was reported in $46 \%$ of children with autism. They also reported that as the number of family members with autoimmune disorders increased from one to three, the risk of autism was greater with an odds ratio that increased from 1.9 to 5.5 , respectively. Thus, this may be an outstanding feature among patients with autism that points to their autoimmune background, with the target in this case being the developing brain.

In our series, the finding of the increased frequency of autoimmune diseases in the mothers of children with autism (25\%) was also in agreement with that of Comi et al. [11] who reported an autoimmune disease in $16 \%$ of the mothers of their studied patients with autism. The high rate of autoimmune diseases in the mothers of the children with autism could also suggest that an autoimmune process exists in the mothers that targets the developing fetus in utero. Although this would be more consistent with the female preponderance in autoimmune disorders, it does not explain the high male-to-female ratio observed in autism [12].

The current study revealed a more significant increase of the frequency of serum antinucleosome-specific antibodies in autistic children with a family history of autoimmunity $(83.3 \%)$ than patients without such a history (22.2\%), $P<0.001$. This implies that in some families, immune dysfunction, perhaps induced by certain environmental triggers, could express itself in the form of autism in one of its offspring. Immune-related genes in the major histocompatibility complex $(\mathrm{MHC})$ may play a central role in the development of autoimmunity in autism. These genes have been associated with some autoimmune diseases such as systemic lupus erythematous and diabetes mellitus. A previous study reported that mothers and their sons had a significantly higher frequency of HLA-DR4 than normal control subjects [50].

To date, a definitive relationship between autism and autoimmunity has not been fully established. On the basis of the preliminary results reported in this study, however, there seems to be a suggestion of evidence in support of autoimmune contributions to the pathophysiology of autism in some cases. Additional investigation designed to expand on these data is warranted.

\section{Conclusions}

Serum levels of antinucleosome-specific antibodies were increased in some autistic children. However, these data should be treated with caution until further investigations are performed with a larger subject population to determine whether these antibodies have a role in the induction of autoimmunity in a subgroup of autistic children. The role of immunotherapy in children with autism should be also studied.

\section{Abbreviations}

CARS: Childhood Autism Rating Scale; IQR: interquartile range; SLE: systemic lupus erythematosus; Th: T helper cells.

\section{Competing interests}

The authors declare that they have no competing interests.

\section{Authors' contributions}

Both authors designed, performed and wrote the research. In addition, both authors read and approved the final manuscript.

\section{Acknowledgements}

This work was financially supported by the King Abdulaziz City for Science and Technology, Riyadh, Saudi Arabia. It was also supported by NPST, Health Research and Studies program at Kind Saud University.

Received: 7 December 2013 Accepted: 10 February 2014

Published: 3 April 2014

\section{References}

1. Cohly HH, Panja A: Immunological findings in autism. Int Rev Neurobiol 2005, 71:317-341.

2. Enstrom AM, Van de Water JA, Ashwood P: Autoimmunity in autism. Curr Opin Investig Drugs 2009, 10:463-473.

3. Singh VK, Lin SX, Yang VC: Serological association of measles virus and human herpesvirus- 6 with brain autoantibodies in autism. Clin Immunol Immunopathol 1998, 89:105-108.

4. Vojdani A, Campbell A, Anyanwu E, Kashanian A, Bock K, Vojdani E: Antibodies to neuron-specific antigens in children with autism: possible cross-reaction with encephalitogenic proteins form milk, Chlamydia pneumoniae and Streptococcus group A. J Neuroimmunol 2002, 129:168-177.

5. Singh VK, Rivas WH: Prevalence of serum antibodies to caudate nucleus in autistic children. Neurosci Lett 2004, 355:53-56.

6. Mostafa GA, El-Sayed ZA, Abd El Aziz MM, El-Sayed MF: Serum anti-myelinassociated glycoprotein antibodies in Egyptian autistic children. J Child Neurol 2008, 23:1413-1418.

7. Mostafa GA, Kitchener N: Serum anti-nuclear antibodies as a marker of autoimmunity in Egyptian autistic children. Pediatr Neurol 2009, 40:107-112.

8. Mostafa GA, El-Hadidi ES, Hewedi DH, Abdou MM: Oxidative stress in Egyptian children with autism: relation to autoimmunity. J Neuroimmunol 2010, 219:114-118. 
9. Mostafa GA, Al-Ayadhi LY: Increased serum levels of anti-ganglioside M1 auto-antibodies in autistic children: relation to the disease severity. J Neuroinflammation 2011, 8:39.

10. Al-Ayadhi LY, Mostafa GA: A lack of association between elevated serum levels of $\mathrm{S} 100 \mathrm{~B}$ protein and autoimmunity in autistic children. J Neuroinflammation 2012, 9:54

11. Comi AM, Zimmerman AW, Frye VH, Law PA, Peeden JN: Familial clustering of autoimmune disorders and evaluation of medical risk factors in autism. J Child Neurol 1999, 14:388-394.

12. Sweeten $\mathrm{TL}$, Bowyer SL, Posey DJ, Halberstadt GM, McDougle CJ: Increased prevalence of familial autoimmunity in probands with pervasive developmental disorders. Pediatrics 2003, 112:420-424.

13. Atladóttir HO, Pedersen MG, Thorsen P, Mortensen PB, Deleuran B, Eaton WW, Parner ET: Association of family history of autoimmune diseases and autism spectrum disorders. Pediatrics 1999, 124:687-694.

14. Mostafa GA, Al Shehab A, Fouad NR: Frequency of $\mathrm{CD} 4^{+} \mathrm{CD} 25^{\text {high }}$ regulatoryT cells in the peripheral blood of Egyptian children with autism. J Child Neurol 2010, 25:328-335.

15. Mostafa GA, Shehab A: The link of C4B null allele to autism and to a family history of autoimmunity in Egyptian autistic children. J Neuroimmunol 2010, 223:115-119.

16. Warren RP, Singh VK, Cole $P$, Odell JD, Pingree CB, Warren $W L$, White E: Increased frequency of the null allele at the complement $C 4 \mathrm{~b}$ locus in autism. Clin Exp Immunol 1991, 83:438-440.

17. Odell D, Maciulis A, Cutler A, Warren L, McMahon WM, Coon H, Stubbs G, Henley K, Torres A: Confirmation of the association of C4B null allele in autism. Human Immunol 2005, 66:140-145.

18. Mostafa GA, Shehab AA, Al-Ayadhi LY: The link between some alleles on human leukocyte antigen system and autism in children. $J$ Neuroimmunol 2013, 255:70-74

19. Vanchu A: Antinuclear antibodies: clinical applications. J Postgrad Med 2000, 46:144-148.

20. Muro Y: Antinuclear antibodies. Autoimmunity 2005, 38:3-9.

21. Amoura Z, Piette JC, Bach JF, Koutouzov S: The key role of nucleosomes in lupus. Arthritis Rheum 1999, 42:833-843.

22. Mohan C, Liu F, Xie C, Williams RC Jr: Anti-subnucleosome reactivities in systemic lupus erythematosus (SLE) patients and their first degree relatives. Clin Exp Immunol 2001, 123:119-126.

23. Amoura Z, Piette JC: Role of the nucleosome in the physiopathology of systemic lupus erythematosus [Abstr]. Ann Med Interne (Paris) 2003, $154: 25-32$.

24. Amoura Z, Koutouzov S, Chabre H, Cacoub P, Amoura I, Musset L, Bach JF, Piette JC: Presence of antinucleosome antibodies in a restricted set of connective tissue diseases: antinucleosome antibodies of lgG3 subclass are markers of renal pathogenicity in systemic lupus erythematosus. Arthritis Rheum 2000, 43:76-84.

25. American Psychiatric Association: Diagnostic and Statistical Manual of Mental Disorders. 4th edition. Washington DC: American Psychiatric Association; 1994.

26. Schopler E, Reichler RJ, Renner BR: The Childhood Autism Rating Scale (CARS), for Diagnostic Screening and Classification in Autism. New York NY: Irvington; 1986

27. Burlingame RW, Rubin RL: Subnucleosome structures as substrates in enzyme- linked immunosorbent assays. J Immunol Methods 1990, 134:187-199.

28. Tumlin JA: Lupus nephritis: novel immunosuppressive modalities and future directions. Semin Nephrol 1999, 19:67-76.

29. Decker P: Nucleosome autoantibodies. Clin Chim Acta 2006, 366(1-2):48-60.

30. Berden JH, Licht R, van Bruggen MC, Tax WJ: Role of nucleosomes for induction and glomerular binding of autoantibodies in lupus nephritis. Curr Opin Nephrol Hypertens 1999, 8:299-306.

31. Bruns A, Blass S, Hausdorf G, Burmester GR, Hiepe F: Nucleosomes are major $\mathrm{T}$ and $\mathrm{B}$ cell autoantigens in systemic lupus erythematosus. Arthritis Rheum 2000, 43:2307-2315.

32. Cozzani E, Drosera M, Gasparini G, Parodi A: Serology of Lupus Erythematosus: Correlation between Immunopathological Features and Clinical Aspects. Autoimmune Dis 2014, : Epub 2014 Feb 6.

33. Burlingame RW, Bocy ML, Starkebaum G, Rubin RL: The central role of chromatin in autoimmune responses to histones and DNA in systemic lupus erythematosus. J Clin Invest 1994, 94:184-192.
34. Chabre H, Amoura Z, Piette JC, Godeau P, Bach JF, Koutouzov S: Presence of nucleosome-restricted antibodies in patients with systemic lupus erythematosus. Arthritis Rheum 1995, 38:1485-1491.

35. Min DJ, Kim SJ, Park SH, Seo YI, Kang HJ, Kim WU, Cho CS, Kim HY: Antinucleosome antibody: significance in lupus patients lacking antidouble stranded DNA antibody. Clin Exp Rheumatol 2002, 20:13-18.

36. Suleiman S, Kamaliah D, Nadeem A, Naing NN, Che Maraina CH: Antinucleosome antibodies as a disease activity marker in patients with systemic lupus erythematosus. Int J Rheum Dis 2009, 12:100-106.

37. Pradhan VD, Patwardhan MM, Ghosh K: Anti-nucleosome antibodies as a disease marker in systemic lupus erythematosus and its correlation with disease activity and other autoantibodies. Indian J Dermatol Venereol Leprol 2010, 76:145-149.

38. Amoura Z, Piette JC, Chabre H, Cacoub P, Papo T, Wechsler B, Bach JF, Koutouzov F: Circulating plasma levels of nucleosomes in patients with systemic lupus erythematosus: Correlation with serum antinucleosome antibody titers and absence of clear association with disease activity. Arthritis Rheum 1997, 40:2217-2225.

39. Lorenz HM, Grunke M, Hieronymus T, Herrmann M, Kuhnel A, Manger B, Kalden JR: In vitro apoptosis and expression of apoptosis-related molecules in lymphocytes from patients with systemic lupus erythematosus and other autoimmune diseases. Arthritis Rheum 1997, 40:306-317.

40. Herrmann M, Voll RE, Zoller OM, Hagenhofer M, Ponner BB, Kalden JR: Impaired phagocytosis of apoptotic cell material by monocyte-derived macrophages from patients with systemic lupus erythematosus. Arthritis Rheum 1998, 41:1241-1250.

41. El-Ansary A, Al-Ayadhi L: Neuroinflammation in autism spectrum disorders. J Neuroinflammation 2012, 9:265.

42. Zakareia FA, Al-Ayadhi LY: Evaluation of plasma soluble fatty acid synthase levels among Saudi autistic children. Relation to disease severity. Neurosciences (Riyadh) 2013, 18:242-247.

43. Chauhan A, Audhya T, Chauhan V: Brain region-specific glutathione redox imbalance in autism. Neurochem Res 2012, 37:1681-1689.

44. Lucarelli S, Frediani T, Zingoni AM, Ferruzzi F, Giardini O, Quintieri F, Barbato M, D'Eufemia P, Cardi E: Food allergy and infantile autism. Panminerva Med 1995, 37:137-141.

45. Jyonouchi $\mathrm{H}$, Sun $\mathrm{S}$, Itokazu N: Innate immunity associated with inflammatory responses and cytokine production against common dietary proteins in patients with autism spectrum disorders. Neuropsychobiology 2002, 46:76-84

46. Mutter J, Naumann J, Schneider R, Walach H, Haley B: Mercury and autism: accelerating evidence? Neuro Endocrinol Lett 2005, 26:439-446.

47. Palmer RF, Blanchard S, Stein Z, Mandell D, Miller C: Environmental mercury release, special education rates, and autism disorder: an ecological study of Texas. Health Place 2006, 12:203-209.

48. Dochniak MJ: Autism spectrum disorders: exogenous protein insult. Med Hypotheses 2007, 89:545-549.

49. Singh VK, Jensen RL: Elevated levels of measles antibodies in children with autism. Pediatr Neurol 2003, 28:292-294.

50. Lee LC, Zachary AA, Leffell MS, Newschaffer CJ, Matteson KJ, Tyler JD, Zimmerman AW: HLA-DR4 in families with autism. Pediatr Neurol 2006, 35:303-307.

\section{doi:10.1186/1742-2094-11-69}

Cite this article as: AL-Ayadhi and Mostafa: Serum antinucleosomespecific antibody as a marker of autoimmunity in children with autism. Journal of Neuroinflammation 2014 11:69. 